\title{
Article
}

\section{Radius of Star-Likeness for Certain Subclasses of Analytic Functions}

\author{
Caihuan Zhang ${ }^{1}$, Mirajul Haq ${ }^{2}$, Nazar Khan ${ }^{3}\left(\mathbb{C}\right.$, Muhammad Arif ${ }^{2}\left(\mathbb{D}\right.$, Khurshid Ahmad $^{4}$ and Bilal Khan ${ }^{5, *(\mathbb{C})}$ \\ 1 Department of Information Technology, Luoyang Normal University, Louyang 471934, China; \\ zhcaihuan@163.com \\ 2 Department of Mathematics, Abdul Wali Khan University Mardan, Mardan 23200, Pakistan; \\ merajkhan054@gmail.com (M.H.); marifmaths@awkum.edu.pk (M.A.) \\ 3 Department of Mathematics, Abbottabad University of Science and Technology, Abbottabad 22010, Pakistan; \\ nazarmaths@gmail.com \\ 4 Government Post Graduate Collage Dargai, Dargai 23060, Pakistan; khurshidahmad410@gmail.com \\ 5 Shanghai Key Laboratory of PMMP, School of Mathematical Sciences, East China Normal University, \\ 500 Dongchuan Road, Shanghai 200241, China \\ * Correspondence: bilalmaths789@gmail.com
}

check for

updates

Citation: Zhang, C.; Haq, M.U.;

Khan, N.; Arif, M.; Ahmad, K.; Khan, B. Radius of Star-Likeness for Certain Subclasses of Analytic Functions. Symmetry 2021, 13, 2448. https:// doi.org/10.3390/sym13122448

Academic Editor: Stanisława Kanas

Received: 16 November 2021 Accepted: 14 December 2021 Published: 19 December 2021

Publisher's Note: MDPI stays neutral with regard to jurisdictional claims in published maps and institutional affiliations.

Copyright: (C) 2021 by the authors. Licensee MDPI, Basel, Switzerland. This article is an open access article distributed under the terms and conditions of the Creative Commons Attribution (CC BY) license (https:// creativecommons.org/licenses/by/ $4.0 /)$.
Abstract: In this paper, we investigate a normalized analytic (symmetric under rotation) function, $f$, in an open unit disk that satisfies the condition $\Re\left(\frac{f(z)}{g(z)}\right)>0$, for some analytic function, $g$, with $\Re\left(\frac{(z+1)^{-2 n}}{z}\right) g(z)>0, \forall n \in \mathbb{N}$. We calculate the radius constants for different classes of analytic functions, including, for example, for the class of star-like functions connected with the exponential functions, i.e., the lemniscate of Bernoulli, the sine function, cardioid functions, the sine hyperbolic inverse function, the Nephroid function, cosine function and parabolic star-like functions. The results obtained are sharp.

Keywords: univalent functions; star-like function; convex function; radius of star-likeness

\section{Introduction and Motivations}

Let by $\mathbb{D}_{r}$, we denote the open unit disk with radius $r$, given by

$$
\mathbb{D}_{r}=\{z: z \in \mathbb{C} \text { and }|z|<r\} .
$$

It can be easily seen that

$$
\mathbb{D}=\mathbb{D}_{1}
$$

Let $\mathcal{H}$ be the family of analytic (symmetric under rotation) functions in

$$
\mathbb{D}=\{z: z \in \mathbb{C} \text { and }|z|<1\}
$$

and $\mathcal{A}_{n} \subset \mathcal{H}$ such that $f \in \mathcal{A}_{n}$ has the series representation:

$$
f(z)=z+\sum_{j=n+1}^{\infty} a_{j} z^{j}
$$

Also, let $\mathfrak{S}$ be the subfamily of $\mathcal{A}_{1}=\mathcal{A}$ containing univalent functions in $\mathbb{D}$. Now, we connect the function $\aleph_{f}(z): \mathbb{D} \rightarrow \mathbb{C}$ defined by $\aleph_{f}(z)=\frac{z f^{\prime}(z)}{f(z)}$ with each $f \in \mathfrak{S}$. The koebe function.

$$
k(z)=z+2 z^{2}+3 z^{3}+\cdots+n z^{n}+\cdots=\sum_{n=2}^{\infty} n z^{n}=\frac{z}{(1-z)^{2}},
$$


which maps $\mathcal{D}$ onto the entire complex plane except for a slit along the negative real axis from $w=-\infty$ to $w=-\frac{1}{4}$, which is star-like but not convex. However it is known that, for $r \leq 2-\sqrt{3}$, the function $k(z)$ maps the disk $\mathbb{D}_{r}=\{z \in \mathbb{C}:|z|<r\}$ onto a convex domain. Indeed for $r \leq 2-\sqrt{3}$ every $f \in \mathfrak{S}$ maps $\mathbb{D}_{r}$ onto a convex region. This number is called the radius of convexity for $\mathfrak{S}$. The radius of star-likeness for functions in the class $\mathfrak{S}$ has been obtained by Grunsky [1] and is given by

$$
\tanh \left(\frac{\pi}{4}\right) \approx 0.6558
$$

For two function classes $Y$ and $\Lambda_{f}$, where the class $\Lambda_{f}$ is characterized by possessing a geometric property $\wp$, we denote by $R_{\Lambda_{f}}(\mathrm{Y})$ the $\Lambda_{f}$ radius of $\mathrm{Y}$, which is the largest number $R$ with $0<r \leq R$ such that $r^{-1} f(r z) \in \Lambda_{f}$ for all $f \in \mathrm{Y}$. Though function theory was first proposed in 1851, it was not until 1916 that Bieberbach's coefficient conjecture made this field a promising new research topic. In 1985, De Branges proved this conjecture. Between 1916 and 1985, many good scholars of the time attempted to prove or reject this theory. As a result, they discovered multiple subfamilies of a class $\mathfrak{S}$ of univalent functions that are associated with different image domains. For example a function $f \in \mathcal{A}$ is said to be close-to-convex if

$$
f^{\prime}(z) \neq 0 \quad \text { and } \quad \Re\left(\frac{f^{\prime}(z)}{g^{\prime}(z)}\right)>0 .
$$

This class was introduced and studied by Kaplan. A function $f \in \mathcal{A}$ with

$$
f(z) \neq 0 \text { and } \Re\left(\frac{f(z)}{g(z)}\right)>0,
$$

for some star-like function, $g$ (not necessarily normalized), was defined by Reade [2]. The work conducted in [3-10] presents some remarkable research in this field and has played a significant role in developing this area of geometric functions theory. Also there has been a lot of research on radius constants for different kinds of functions that are defined by their ratio to a certain function $g$. Mac-Gregor [11,12] obtained the radius of star-likeness for the class of functions $f \in \mathrm{Y}$ satisfying

$$
\Re\left(\frac{f(z)}{g(z)}\right)>0 \text { or }\left|\frac{f(z)}{g(z)}-1\right|<1 \quad(z \in \mathbb{D}) .
$$

Ratti [13] established the radius for the function $f$ satisfying

$$
\left|\frac{f^{\prime}(z)}{g^{\prime}(z)}-1\right|<1 \quad(z \in \mathbb{D}),
$$

where $g$ belongs to certain classes of analytic functions. Lecko and Sim [14] discovered that certain functions satisfy the conditions $\Re\left(1-z^{2}\right) \frac{f(z)}{z}>0$ and $\Re(1-z)^{2} f(z) / z>0$ by considering the star-like functions $\frac{z}{1-z^{2}}$ and $\frac{z}{(1-z)^{2}}$. They have also determined certain sharp coefficient estimates. A function $\chi_{1}$ (holomorphic function) is subordinate to another function $\chi_{2}$, written as

$$
\chi_{1} \prec \chi_{2},
$$

if there is an analytic function $v$ with

$$
|v(z)| \leq|z| \quad \text { and } \quad v(0)=0,
$$

such that

$$
\chi_{1}(z)=\chi_{2}(v(z))
$$


If $\chi_{2}$ is univalent then

$$
\chi_{1} \prec \chi_{2} \Longleftrightarrow \chi_{1}(0)=\chi_{2}(0) \text { and } \chi_{1}(\mathbb{D}) \subseteq \chi_{2}(\mathbb{D}) .
$$

We now have the functions $\varphi_{j}: \mathbb{D} \rightarrow \mathbb{C}$, given by

$$
\begin{gathered}
\varphi_{1}(z)=\sqrt{1+z}, \quad \varphi_{2}(z)=1+z-\frac{z^{3}}{3}, \quad \varphi_{3}(z)=\cos z, \\
\varphi_{4}(z)=e^{z}, \quad \varphi_{5}(z)=1+\frac{4}{3} z+\frac{2}{3} z^{2}, \quad \varphi_{6}(z)=1+\sin z, \\
\varphi_{7}(z)=z+\sqrt{z^{2}+1}, \quad \varphi_{8}(z)=1+\sinh ^{-1}(z) .
\end{gathered}
$$

For $\varphi=\varphi_{j}(j=1,2, \cdots 8)$ the class $S^{*}(\varphi)=\left\{f \in \mathcal{A}\right.$ : $\left.\aleph_{f}(z) \prec \phi(z)\right\}$ respectively becomes $\mathcal{S}_{L}^{*}, \mathcal{S}_{\mathrm{Ne}}^{*}, \mathcal{S}_{\mathrm{cos}}^{*}, \mathcal{S}_{e}^{*}, \mathcal{S}_{c}^{*}, \mathcal{S}_{\mathrm{sin}^{\prime}}^{*} \mathcal{S}_{p}^{*}$ and $\mathcal{S}_{\text {sinh }^{-1}}^{*}$. These classes were extensionally studied in [15-22]. Many well-known mathematicians found radius constants for several classes of functions $f \in \mathrm{Y}$ that, defined by their ratio to a certain function $g$, meet one of the following conditions:

I $\quad \Re\left(\frac{f(z)}{g(z)}\right)>0$ where $\Re\left(\frac{g(z)}{z}\right)>0$ or $\Re\left(\frac{g(z)}{z}\right)>\frac{1}{2}$.

II $\left|\frac{f(z)}{g(z)}-1\right|<1$ where $\Re\left(\frac{g(z)}{z}\right)>0$.

III $\left|\frac{f^{\prime}(z)}{g^{\prime}(z)}-1\right|<1$ where $\Re g^{\prime}(z)>0$ or $g$ is convex.

Motivated by the above studies we introduced the following class

$\mathrm{Y}_{n}=\left\{f \in \mathcal{A}: \Re\left(\frac{f(z)}{g(z)}\right)>0, \Re \frac{g(z)}{z(1+z)^{2 n}}>0, \forall n \in \mathbb{N}\right.$ and for some $\left.g \in \mathcal{A}\right\}$.

If the mapping $f_{n}: \mathbb{C} \rightarrow \mathbb{D}$ is defined by

$$
f_{n}(z)=\frac{z(1+z)^{2 n+2}}{(1-z)^{2}} \quad(n \in \mathbb{N}) .
$$

Then it follows that $f_{n}(z)$ belong to the class $Y_{n}$ and so class $Y_{n}$ is non empty, also the function $f_{n}$ satisfy the conditions

$$
\Re\left(\frac{f_{n}(z)}{g(z)}\right)>0, \Re \frac{g(z)}{z(1+z)^{2 n}}>0, \forall n \in \mathbb{N} \text { and for some } g \in \mathcal{A},
$$

with $g=g_{n}$, where

$$
g_{n}(z)=\frac{z(1+z)^{2 n+1}}{1-z} \quad(\forall z \in \mathbb{D} \text { and } n \in \mathbb{N}) .
$$

The functions in the class $Y_{n}$ need not to be univalent. Indeed

$$
R_{S}\left(\mathrm{Y}_{n}\right) \approx \frac{2}{2 n+4+\sqrt{(2 n+4)^{2}+4(2 n+1)}}, \forall n \in \mathbb{N},
$$

which is the radius of univalence, the least positive root of the polynomial

$$
p_{n}(r)=1-(2 n+1) r^{2}-(2 n+4) r .
$$

The derivative of $f_{n}$ is given by

$$
f_{n}^{\prime}(z)=-\frac{1}{(z-1)^{3}}(z+1)^{2 n+1}\left(4 z-2 n z^{2}+2 n z-z^{2}+1\right)
$$


and vanishes at $z=-R_{s}\left(\mathrm{Y}_{n}\right)$; for all $n \in \mathbb{N}$. For $n=1,2,3$ the class $\mathrm{Y}_{n}$ reduces to the following classes

$$
\begin{aligned}
& \mathrm{Y}_{1}=\left\{f \in \mathcal{A}: \Re\left(\frac{f(z)}{g(z)}\right)>0, \Re \frac{g(z)}{z(1+z)^{2}}>0, \text { for some } g \in \mathcal{A}\right\}, \\
& \mathrm{Y}_{2}=\left\{f \in \mathcal{A}: \Re\left(\frac{f(z)}{g(z)}\right)>0, \Re \frac{g(z)}{z(1+z)^{4}}>0, \text { for some } g \in \mathcal{A}\right\}, \\
& \mathrm{Y}_{3}=\left\{f \in \mathcal{A}: \Re\left(\frac{f(z)}{g(z)}\right)>0, \Re \frac{g(z)}{z(1+z)^{6}}>0, \text { for some } g \in \mathcal{A}\right\} .
\end{aligned}
$$

For $n=1$ the functions defined in (4) and (5) reducesto the following functions

$$
f_{1}(z)=\frac{z(1+z)^{4}}{(1-z)^{2}}, \quad g_{1}(z)=\frac{z(1+z)^{3}}{1-z}
$$

and satisfy

$$
\Re\left(\frac{f_{1}(z)}{g_{1}(z)}\right)=\Re \frac{g_{1}(z)}{z(1+z)^{2}}=\Re\left(\frac{1+z}{1-z}\right)>0 .
$$

For $n=2$ the functions defined in (4) and (5) reduce to the following functions

$$
f_{2}(z)=\frac{z(1+z)^{6}}{(1-z)^{2}}, \quad g_{2}(z)=\frac{z(1+z)^{5}}{1-z}
$$

and satisfy

$$
\Re\left(\frac{f_{2}(z)}{g_{2}(z)}\right)=\Re \frac{g_{2}(z)}{z(1+z)^{4}}=\Re\left(\frac{1+z}{1-z}\right)>0 .
$$

For $n=3$ the functions defined in (4) and (5) reduce to the following functions

$$
f_{3}(z)=\frac{z(1+z)^{8}}{(1-z)^{2}}, \quad g_{3}(z)=\frac{z(1+z)^{7}}{1-z}
$$

and satisfy

$$
\Re\left(\frac{f_{2}(z)}{g_{2}(z)}\right)=\Re \frac{g_{2}(z)}{z(1+z)^{6}}=\Re\left(\frac{1+z}{1-z}\right)>0 .
$$

For $0<\alpha \leq 1$. Let $\mathcal{P}(\alpha)$ denote the class of functions $p(z)=1+b_{1} z+\cdots$ satisfying

$$
\Re(p(z))>\alpha \quad(\forall z \in \mathbb{D}) .
$$

We note that

$$
\mathcal{P}(0)=\mathcal{P},
$$

where $\mathcal{P}$ is a known class of functions with a positive, real part, or the class of Caratheodory functions. If $p \in \mathcal{P}(\alpha)$, then

$$
\left|\frac{z p^{\prime}(z)}{p(z)}\right| \leq \frac{2(1-\alpha) r}{(1-r)(1+(1-2 \alpha) r)}
$$

In this paper, we aim to calculate radius constants for certain classes of star-like functions related with different types of domains, including, for example, star-like functions connected with the exponential functions, the lemniscate of Bernoulli, the sine function, cardioid functins, the sine hyperbolic inverse function, the Nephroid function, cosine functions and parabolic star-like functions. The results presented in this paper are sharp. Additionally, by assigning different values to some perpetrators we obtain some interesting special cases of our main results. 


\section{Radius Problems}

To solve radius problems in this article, we find a disk such that it contained the image of $\mathbb{D}_{r}$ under the mapping $\frac{z f^{\prime}(z)}{f(z)}$ for the function $f$ belonging to the class $Y_{n}$. Additionally, for the radius constants of different subclasses of star-like functions, we take the function $w(z)=\frac{1}{1+z}$, which maps $\mathbb{D}_{r}$ onto the following disk

$$
\left|\frac{1}{1+z}-\frac{1}{1-r^{2}}\right| \leq \frac{r}{1-r^{2}} .
$$

Theorem 1. For the functions class $Y_{n}$, we have the following results

1. The $\mathcal{S}^{*}(\alpha)$ radius for $\mathrm{Y}_{n}$ is

$$
R_{\mathcal{S}^{*}(\alpha)}=\frac{2(1-\alpha)}{2 n+4+\sqrt{(2 n+4)^{2}+4(1-\alpha)(2 n+1-\alpha)}} .
$$

2. The $\mathcal{S}_{L}^{*}$ radius for $\mathrm{Y}_{n}$ is

$$
R_{\mathcal{S}_{L}^{*}}=\frac{2(\sqrt{2}-1)}{2 n+4+\sqrt{(2 n+4)^{2}-4(1-\sqrt{2})(\sqrt{2}-1-2 n)}} .
$$

3. The $\mathcal{S}_{p}^{*}$ radius for $Y_{n}$ is

$$
R_{\mathcal{S}_{p}^{*}}=\frac{2}{4 n+8+\sqrt{(4 n+8)^{2}+4(4 n+1)}} .
$$

4. The $\mathcal{S}_{e}^{*}$ radius for $Y_{n}$ is

$$
R_{\mathcal{S}_{e}^{*}}=\frac{2(e-1)}{(2 n+4) e+\sqrt{(2 n+4)^{2} e^{2}+4(2 n e+e-1)(e-1)}} .
$$

5. The $\mathcal{S}_{\mathcal{c}}^{*}$ radius for $\mathrm{Y}_{n}$ is

$$
R_{\mathcal{S}_{c}^{*}}=\frac{4}{6 n+12+\sqrt{(6 n+12)^{2}+8(6 n+2)}} .
$$

6. The $\mathcal{S}_{\text {sin }}^{*}$ radius for $Y_{n}$ is

$$
R_{\mathcal{S}_{\text {sin }}^{*}}=\frac{2 \sin (1)}{2 n+4+\sqrt{(2 n+4)^{2}+4 \sin (1)(2 n+\sin (1))}} .
$$

7. The $\mathcal{S}_{\sinh ^{-1}}^{*}$ radius for $\mathrm{Y}_{n}$ is

$$
R_{\mathcal{S}_{\text {sinh }^{*}}}=\frac{2 \sinh ^{-1}(1)}{2 n+4+\sqrt{(2 n+4)^{2}+4 \sinh ^{-1}(1)\left(2 n+\sinh ^{-1}(1)\right)}} .
$$

8. The $\mathcal{S}_{N_{e}}^{*}$ radius for $\mathrm{Y}_{n}$ is

$$
R_{\mathcal{S}_{N_{e}}^{*}}=\frac{2}{3 n+6+\sqrt{(3 n+6)^{2}+4(3 n+1)}}=R_{\mathcal{S}_{c}^{*}} .
$$


9. The $\mathcal{S}_{\mathrm{cos}}^{*}$ radius for $\mathrm{Y}_{n}$ is

$$
R_{\mathcal{S}_{\mathrm{cos}}^{*}}=\frac{2(1-\cos (1))}{2 n+4+\sqrt{(2 n+4)^{2}+4(2 n+1)(1-\cos 1)}} .
$$

Proof. Let $f \in \mathrm{Y}_{n}$. We choose the function $g: \mathbb{D} \rightarrow \mathbb{C}$ satisfying the following inequalities

$$
\Re\left(\frac{1}{z(1+z)^{2 n}}\right) g(z)>0 \text { and } \Re\left(\frac{f(z)}{g(z)}\right)>0, \quad(\forall z \in \mathbb{D} \text { and } n \in \mathbb{N}) \text {. }
$$

We also choose the functions $p_{1}, p_{2}: \mathbb{D} \rightarrow \mathbb{C}$ by

$$
p_{1}(z)=\left(\frac{1}{z(1+z)^{2 n}}\right) g(z) \quad \text { and } \quad p_{2}(z)=\frac{f(z)}{g(z)}
$$

From $(11)$ and $(12) p_{1}(z), p_{2}(z) \in \mathcal{P}$ and we define $f(z)$ by

$$
f(z)=\frac{z p_{1}(z) p_{2}(z)}{(1+z)^{-2 n}}
$$

The logarithmic differentiation of (13) gives

$$
\begin{aligned}
\frac{z f^{\prime}(z)}{f(z)} & =1+\frac{z p_{1}^{\prime}(z)}{p_{1}(z)}+\frac{z p_{2}^{\prime}(z)}{p_{2}(z)}+\frac{2 n z}{1+z} \\
& =1+\frac{z p_{1}^{\prime}(z)}{p_{1}(z)}+\frac{z p_{2}^{\prime}(z)}{p_{2}(z)}+2 n-\frac{2 n}{1+z} \\
& =2 n+1+\frac{z p_{1}^{\prime}(z)}{p_{1}(z)}+\frac{z p_{2}^{\prime}(z)}{p_{2}(z)}-\frac{2 n}{1+z} .
\end{aligned}
$$

Using (9), (10) and (14) we get

$$
\left|\frac{z f^{\prime}(z)}{f(z)}-\frac{1-(2 n+1) r^{2}}{1-r^{2}}\right| \leq \frac{(2 n+4) r}{1-r^{2}}
$$

From (15), we have

$$
\Re\left(\frac{z f^{\prime}(z)}{f(z)}\right) \geq \frac{1-(2 n+1) r^{2}-(2 n+4) r}{1-r^{2}} \geq 0 \quad\left(r \leq \kappa_{n}\right),
$$

where

$$
\kappa_{n}=\frac{2}{2 n+4+\sqrt{(2 n+4)^{2}+4(2 n+1)}} .
$$

Hence all of the radii we estimate will be less than $\kappa_{n}$.

1. The number $r=R_{S *(\alpha)}$ is the zero of the polynomial $(2 n+1-\alpha) r^{2}+(2 n+4) r-$ $(1-\alpha)=0$ in the interval $[0,1]$ and, so, for $0<r \leq R_{S^{*}(\alpha)}$, we have from (16) that

$$
\Re\left(\frac{z f^{\prime}(z)}{f(z)}\right) \geq \frac{1-(2 n+1) r^{2}-(2 n+4) r}{1-r^{2}} \geq \alpha .
$$

For the function $f_{n} \in \mathrm{Y}_{n}$, given by (4), we have

$$
\frac{z f_{n}^{\prime}(z)}{f_{n}(z)}=\frac{1-(2 n+1) r^{2}-(2 n+4) r}{1-r^{2}}=\alpha \quad\left(z=-R_{S *(\alpha)}\right),
$$

which shows that this result is sharp. 
2. Let $R=R_{S_{L}^{*}}$ be the root (smallest positive) of the polynomial

$$
1-(2 n+1) R^{2}+(2 n+4) R=\sqrt{2}\left(1-R^{2}\right) .
$$

Let

$$
\Xi_{1}(r)=\frac{(2 n+4) r}{1-r^{2}}+\frac{1-(2 n+1) r^{2}}{1-r^{2}}=\frac{1-(2 n+1) r^{2}+(2 n+4) r}{1-r^{2}},
$$

clearly the values of the function $\Xi_{1}(r)$ increases for every point on that interval $[0,1)$, for all $n \in \mathbb{N}$. As a result, for $0 \leq r<R, \Xi_{1}(r)<\Xi_{1}(R)=\sqrt{2}$ and so, we have

$$
\frac{(2 n+4) r}{1-r^{2}} \leq \sqrt{2}-\frac{1-(2 n+1) r^{2}}{1-r^{2}}, \text { for } 0 \leq r<R .
$$

From (15) and (17) we achieve

$$
\left|\frac{z f^{\prime}(z)}{f(z)}-\frac{1-(2 n+1) r^{2}}{1-r^{2}}\right| \leq \sqrt{2}-\frac{1-(2 n+1) r^{2}}{1-r^{2}} .
$$

For $0 \leq r<R$, the function $\Lambda(r)=\frac{1-(2 n+1) r^{2}}{1-r^{2}}$ (center of the above disk) shows that $\Lambda^{\prime}(r)<0$ for every point on that interval $([0,1], \forall n \in \mathbb{N})$, and lies in the following interval

$$
[\Lambda(R), 1] \subset(\Lambda(0.1), 1] \approx\left(1.0-2.0202 \times 10^{-2} n, 1\right] \subset\left[\frac{2 \sqrt{2}}{3}, \sqrt{2}\right)
$$

by [23], the disk $|w-a|<r_{a}$ is contained in the lemniscate region $\left|w^{2}-1\right|<1$ where

$$
r_{a}= \begin{cases}\sqrt{1-a^{2}}-\left(1-a^{2}\right)^{\frac{1}{2}} & \left(0<a \leq \frac{2 \sqrt{2}}{3}\right) \\ \sqrt{2}-a & \left(\frac{2 \sqrt{2}}{3} \leq a<\sqrt{2}\right) .\end{cases}
$$

Therefore for $0 \leq r<R$, we get

$$
\left|\left(\frac{z f^{\prime}(z)}{f(z)}\right)^{2}-1\right|<1
$$

For the functions $f_{n} \in \mathrm{Y}_{n}$, given by (4), we have

$$
\frac{z f_{n}^{\prime}(z)}{f_{n}(z)}=\frac{1-(2 n+1) R^{2}+(2 n+4) R}{\left(1-R^{2}\right)}=\sqrt{2}, \text { at } z=R,
$$

and hence

$$
\left|\left(\frac{z f_{n}^{\prime}(z)}{f_{n}(z)}\right)^{2}-1\right|=1
$$

3. The number $R=R_{S_{\tilde{p}}^{*}}$ is the root (smallest positive) of the polynomial

$$
1-(2 n+1) R^{2}-(2 n+4) R=\frac{1}{2}\left(1-R^{2}\right) .
$$

Let

$$
\Xi_{2}(r)=\frac{-(2 n+4) r}{1-r^{2}}+\frac{1-(2 n+1) r^{2}}{1-r^{2}}=\frac{1-(2 n+1) r^{2}-(2 n+4) r}{1-r^{2}},
$$


clearly the values of the function $\Xi_{2}(r)$ decrease for every point on that interval $[0,1)$, for all $n \in \mathbb{N}$. As a result, for $0 \leq r<R, \Xi_{2}(r)>\Xi_{2}(R)=1 / 2$ and hence we have

$$
\frac{(2 n+4) r}{1-r^{2}}<\frac{1-(2 n+1) r^{2}}{1-r^{2}}-\frac{1}{2}, 0 \leq r<R .
$$

From (15) and (23)

$$
\left|\frac{z f^{\prime}(z)}{f(z)}-\frac{1-(2 n+1) r^{2}}{1-r^{2}}\right|<\frac{1-(2 n+1) r^{2}}{1-r^{2}}-\frac{1}{2} \quad(|z| \leq r) .
$$

For $0 \leq r<R$, the function $\Lambda(r)=\frac{1-(2 n+1) r^{2}}{1-r^{2}}$ shows that $\Lambda^{\prime}(r)<0$ for every point on that interval $([0,1], \forall n \in \mathbb{N})$, and lies in the following interval

$$
[\Lambda(R), 1] \subset[\Lambda(.2), 1] \approx\left[1.0-2.0202 \times 10^{-2} n, 1\right] \subset\left(\frac{1}{2}, \frac{3}{2}\right)
$$

By [24], we have

$$
\Omega=\left\{|w-a|<r_{a}\right\} \subset\{w \in \mathbb{C}:|w-1|<\Re w\} .
$$

where $r_{a}$ is given by

$$
r_{a}= \begin{cases}a-\frac{1}{2} & \left(\frac{1}{2}<a<\frac{3}{2}\right) \\ \sqrt{2}-a & \left(a>\frac{3}{2}\right) .\end{cases}
$$

Therefore, we get

$$
\left|\frac{z f^{\prime}(z)}{f(z)}-1\right|<\Re\left(\frac{z f^{\prime}(z)}{f(z)}\right) \quad(|z| \leq r) .
$$

The result is sharp for the function $f_{n}$ defined in (4), satisfies, at $z=-R$

$$
\frac{z f_{n}^{\prime}(z)}{f_{n}(z)}=\frac{1-(2 n+1) R^{2}-(2 n+4) R}{1-R^{2}}=\frac{1}{2}
$$

therefore

$$
\frac{z f_{n}^{\prime}(z)}{f_{n}(z)}-1=\frac{1}{2}=\Re\left(\frac{z f_{n}^{\prime}(z)}{f_{n}(z)}\right) .
$$

4. The number $R=R_{S_{e}^{*}}$ is the root (least positive) of the polynomial

$$
1-(2 n+1) R^{2}-(2 n+4) R=e^{-1}\left(1-R^{2}\right),
$$

clearly the values of the function

$$
\Xi_{2}(r)=\frac{-(2 n+4) r}{1-r^{2}}+\frac{1-(2 n+1) r^{2}}{1-r^{2}}=\frac{1-(2 n+1) r^{2}-(2 n+4) r}{1-r^{2}},
$$

decreases for every point on that interval $[0,1)$, for all $n \in \mathbb{N}$. As a result, for $0 \leq r<R$, $\Xi_{2}(r)>\Xi_{2}(R)=1 / e$ and hence we have

$$
\frac{(2 n+4) r}{1-r^{2}}<\frac{1-(2 n+1) r^{2}}{1-r^{2}}-\frac{1}{e} .
$$


From (15) and (26), we have

$$
\left|\frac{z f^{\prime}(z)}{f(z)}-\frac{1-(2 n+1) r^{2}}{1-r^{2}}\right| \leq \frac{1-(2 n+1) r^{2}}{1-r^{2}}-\frac{1}{e}, \quad(|z| \leq r)
$$

For $0 \leq r<R$, the function $\Lambda(r)=\frac{1-(2 n+1) r^{2}}{1-r^{2}}$ shows that $\Lambda^{\prime}(r)<0$ for every point on that interval $([0,1], \forall n \in \mathbb{N})$, and lies in the following interval

$$
[\Lambda(R), 1] \subset[\Lambda(.2), 1] \approx\left[1.0-2.0202 \times 10^{-2} n, 1\right] \subset\left(\frac{1}{e^{\prime}}, \frac{e+e^{-1}}{2}\right)
$$

By [25] we have

$$
\left\{w \in \mathbb{C}:|w-a|<r_{a}\right\} \subset\{w \in \mathbb{C}:|\log w(z)|<1\}
$$

where

$$
r_{a}=\left\{\begin{array}{cc}
a-\frac{1}{e}, & \frac{1}{e}<a \leq \frac{e+e^{-1}}{2} \\
e-a & \frac{e+e^{-1}}{2}<a \leq e .
\end{array}\right.
$$

Hence for $0 \leq r<R$ we have

$$
\left|\log \left(\frac{z f^{\prime}(z)}{f(z)}\right)\right|<1 \quad(|z| \leq r) .
$$

The result is sharp for the function $f_{n}$ defined in (4), satisfies at $z=-R$

$$
\frac{z f_{n}^{\prime}(z)}{f_{n}(z)}=\frac{1-(2 n+4) R-(2 n+1) R^{2}}{1-R^{2}}=\frac{1}{e}
$$

and so

$$
\left|\log \frac{z f_{n}^{\prime}(z)}{f_{n}(z)}\right|=\left|\log \left(\frac{1-(2 n+4) R-(2 n+1) R^{2}}{1-R^{2}}\right)\right|=1 .
$$

5. The number $R=R_{S_{c}^{*}}$ is the least positive root of the equation

$$
1-(2 n+1) R^{2}-(2 n+4) R=\frac{1}{3}\left(1-R^{2}\right),
$$

clearly the values of the function

$$
\Xi_{2}(r)=\frac{-(2 n+4) r}{1-r^{2}}+\frac{1-(2 n+1) r^{2}}{1-r^{2}}=\frac{1-(2 n+1) r^{2}-(2 n+4) r}{1-r^{2}},
$$

decreases for every point on that interval $[0,1)$, for all $n \in \mathbb{N}$. As a result, for $0 \leq r<R$, $\Xi_{2}(r)>\Xi_{2}(R)=1 / 3$ and hence we have

$$
\frac{(2 n+4) r}{1-r^{2}}<\frac{1-(2 n+1) r^{2}}{1-r^{2}}-\frac{1}{3}
$$

From (15) and (30), we have

$$
\left|\frac{z f^{\prime}(z)}{f(z)}-\frac{1-(2 n+1) r^{2}}{1-r^{2}}\right| \leq \frac{1-(2 n+1) r^{2}}{1-r^{2}}-\frac{1}{3}, \quad(|z| \leq r) .
$$

For $0 \leq r<R$, the function $\Lambda(r)=\frac{1-(2 n+1) r^{2}}{1-r^{2}}$ showing that $\Lambda^{\prime}(r)<0$ for every point on that interval $([0,1], \forall n \in \mathbb{N})$, and lies in the following interval

$$
[\Lambda(R), 1] \subset[\Lambda(.2), 1] \approx\left[1.0-2.0202 \times 10^{-2} n, 1\right] \subset\left(\frac{1}{3}, \frac{5}{3}\right)
$$


It follows from [18] that

$$
\left\{w \in \mathbb{C}:|w-a|<r_{a}\right\} \subset \Omega_{c},
$$

where

$$
r_{a}=\left\{\begin{array}{cc}
a-\frac{1}{3}, & \frac{1}{3}<a \leq \frac{5}{3} \\
3-a & \frac{5}{3}<a \leq 3 .
\end{array}\right.
$$

and $\Omega_{c}$ is the region bounded by cardioid

$$
\left\{x+i y:\left(9 x^{2}+9 y^{2}-18 x+5\right)^{2}-16\left(9 x^{2}+9 y^{2}-6 x+1\right)\right\} .
$$

Hence

$$
\mathfrak{S}_{f}\left(\mathbb{D}_{r}\right) \subset \varphi_{5}(\mathbb{D}) \quad(0 \leq r<R) .
$$

The result is sharp for the function $f_{n}$ defined in (4). At $z=-R$

$$
\left|\frac{z f_{n}^{\prime}(z)}{f_{n}(z)}\right|=\left|\left(\frac{1-(2 n+4) R-(2 n+1) R^{2}}{1-R^{2}}\right)\right|=\frac{1}{3}=\varphi_{5}(-1) \in \partial_{c} \varphi_{5}(\mathbb{D}) .
$$

6. The number $R=R_{S_{\text {sin }}^{*}}$ is the root (least positive) of the equation

$$
1-(2 n+1) R^{2}-(2 n+4) R=(1-\sin (1))\left(1-R^{2}\right),
$$

Clearly, the values of the function

$$
\Xi_{2}(r)=\frac{-(2 n+4) r}{1-r^{2}}+\frac{1-(2 n+1) r^{2}}{1-r^{2}}=\frac{1-(2 n+1) r^{2}-(2 n+4) r}{1-r^{2}},
$$

decrease for every point on that interval $[0,1)$, for all $n \in \mathbb{N}$. As a result, for $0 \leq r<R$, $\Xi_{2}(r)>\Xi_{2}(R)=1 / 1-\sin (1)$ and hence we have

$$
\frac{(2 n+4) r}{1-r^{2}}<\frac{1-(2 n+1) r^{2}}{1-r^{2}}+\sin (1)-1 .
$$

From (15) and (34), we have

$$
\left|\frac{z f^{\prime}(z)}{f(z)}-\frac{1-(2 n+1) r^{2}}{1-r^{2}}\right| \leq \frac{1-(2 n+1) r^{2}}{1-r^{2}}+\sin (1)-1, \quad(|z| \leq r) .
$$

For $0 \leq r<R$, the function $\Lambda(r)=\frac{1-(2 n+1) r^{2}}{1-r^{2}}$ shows that $\Lambda^{\prime}(r)<0$ for every point on that interval $([0,1], \forall n \in \mathbb{N})$ and lies in the following interval

$$
[\Lambda(R), 1] \subset[\Lambda(.2), 1] \approx\left[1.0-2.0202 \times 10^{-2} n, 1\right] \subset(1-\sin (1), 1+\sin (1)) .
$$

It follows from [20] that

$$
\left\{w \in \mathbb{C}:|w-a|<r_{a}\right\} \subset \Omega_{s} \subset\{w \in \mathbb{C}:|w(z)-1|<\sinh (1)\},
$$

where $\Omega_{s}$ is the image of $\mathbb{D}$ under the mapping $\vartheta_{0}(z)=1+\sin z$ and $r_{a}$ is given by

$$
r_{a}= \begin{cases}a+\sin 1-1 & (1-\sin 1<a \leq 1) \\ 1+\sin 1-a & (1<a \leq 1+\sin 1) .\end{cases}
$$

Hence

$$
\mathfrak{S}_{f}\left(\mathbb{D}_{r}\right) \subset \varphi_{6}(\mathbb{D}) \quad(0 \leq r<R) .
$$


The result is sharp for the function $f_{n}$ defined in (4). At $z=-R$

$$
\begin{aligned}
\left|\frac{z f_{n}^{\prime}(z)}{f_{n}(z)}\right| & =\left|\left(\frac{1-(2 n+4) R-(2 n+1) R^{2}}{1-R^{2}}\right)\right| \\
& =1-\sin (1)=\varphi_{6}(-1) \in \partial_{c} \varphi_{6}(\mathbb{D}) .
\end{aligned}
$$

7. The number $R=R_{S_{\text {sinh }^{*}}^{*}}$ is the root (least positive) of the polynomial

$$
1-(2 n+1) R^{2}-(2 n+4) R=\left(1-\sinh ^{-1}(1)\right)\left(1-R^{2}\right),
$$

Clearly, the values of the function

$$
\Xi_{2}(r)=\frac{-(2 n+4) r}{1-r^{2}}+\frac{1-(2 n+1) r^{2}}{1-r^{2}}=\frac{1-(2 n+1) r^{2}-(2 n+4) r}{1-r^{2}},
$$

decrease for every point on that interval $[0,1)$, for all $n \in \mathbb{N}$. As a result, for $0 \leq r<R$, $\Xi_{2}(r)>\Xi_{2}(R)=1 / 1-\sinh ^{-1}(1)$ and hence we have

$$
\frac{(2 n+4) r}{1-r^{2}}<\frac{1-(2 n+1) r^{2}}{1-r^{2}}+\sinh ^{-1}(1)-1 \text {. }
$$

From (15) and (38), we have

$$
\left|\frac{z f^{\prime}(z)}{f(z)}-\frac{1-(2 n+1) r^{2}}{1-r^{2}}\right| \leq \frac{1-(2 n+1) r^{2}}{1-r^{2}}+\sinh ^{-1}(1)-1, \quad(|z| \leq r) .
$$

For $0 \leq r<R$, the function $\Lambda(r)=\frac{1-(2 n+1) r^{2}}{1-r^{2}}$ shows that $\Lambda^{\prime}(r)<0$ for every point on that interval $([0,1], \forall n \in \mathbb{N})$, and lies in the following interval

$$
[\Lambda(R), 1] \subset[\Lambda(.2), 1] \approx\left[1.0-2.0202 \times 10^{-2} n, 1\right] \subset\left(1-\sinh ^{-1}(1), 1+\sinh ^{-1}(1)\right) .
$$

By [26], we have

$$
\left\{w \in \mathbb{C}:|w-a|<r_{a}\right\} \subset \Omega_{\sinh ^{-1}} \subset\left\{w \in \mathbb{C}:|w(z)-1|<\frac{\pi}{2}\right\},
$$

where $\Omega_{\sinh ^{-1}}$ is the image of $\mathbb{D}$ under the mapping $\Psi_{0}(z)=1+\sinh ^{-1} z$ and $r_{a}$ is given by

$$
r_{a}= \begin{cases}a-\left(1-\sinh ^{-1}(1)\right) & \left(1-\sinh ^{-1}(1)<a \leq 1\right) \\ 1+\sinh ^{-1}(1)-a & \left(1<a \leq 1+\sinh ^{-1}(1)\right) .\end{cases}
$$

Hence

$$
\mathfrak{S}_{f}\left(\mathbb{D}_{r}\right) \subset \varphi_{8}(\mathbb{D}) \quad(0 \leq r<R) .
$$

The result is sharp for the function $f_{n}$ defined in (4). At $z=-R$

$$
\left|\frac{z f_{n}^{\prime}(z)}{f_{n}(z)}\right|=\left|\left(\frac{1-(2 n+4) R-(2 n+1) R^{2}}{1-R^{2}}\right)\right|=1-\sinh ^{-1}(1)=\varphi_{8}(-1) \in \partial_{c} \varphi_{8}(\mathbb{D}) .
$$

8. The number $R=R_{S_{N e}^{*}}$ is the root (least positive) of the polynomial

$$
1-(2 n+1) R^{2}-(2 n+4) R=\frac{1}{3}\left(1-R^{2}\right),
$$


Clearly, the values of the function

$$
\Xi_{2}(r)=\frac{-(2 n+4) r}{1-r^{2}}+\frac{1-(2 n+1) r^{2}}{1-r^{2}}=\frac{1-(2 n+1) r^{2}-(2 n+4) r}{1-r^{2}},
$$

decrease for every point on that interval $[0,1)$, for all $n \in \mathbb{N}$. As a result, for $0 \leq r<R$, $\Xi_{2}(r)>\Xi_{2}(R)=1 / 3$ and hence, we have

$$
\frac{(2 n+4) r}{1-r^{2}}<\frac{1-(2 n+1) r^{2}}{1-r^{2}}-\frac{1}{3} .
$$

From (15) and (42), we have

$$
\left|\frac{z f^{\prime}(z)}{f(z)}-\frac{1-(2 n+1) r^{2}}{1-r^{2}}\right| \leq \frac{1-(2 n+1) r^{2}}{1-r^{2}}-\frac{1}{3}, \quad(|z| \leq r) .
$$

For $0 \leq r<R$, the function $\Lambda(r)=\frac{1-(2 n+1) r^{2}}{1-r^{2}}$ shows that $\Lambda^{\prime}(r)<0$ for every point on that interval $([0,1], \forall n \in \mathbb{N})$, and lies in the following interval

$$
[\Lambda(R), 1] \subset[\Lambda(.2), 1] \approx\left[1.0-2.0202 \times 10^{-2} n, 1\right] \subset\left(\frac{1}{3}, 1\right] .
$$

By [19] we have

$$
\left\{w \in \mathbb{C}:|w-a|<r_{a}\right\} \subset \Omega_{N e},
$$

where $\Omega_{N e}$ is the region bounded by the nephroid and $r_{a}$ is given by

$$
r_{a}= \begin{cases}a-\frac{1}{3}, & \left(\frac{1}{3}<a \leq 1\right) \\ \frac{5}{3}-a & \left(1 \leq a \leq \frac{5}{3}\right) .\end{cases}
$$

Hence

$$
\mathfrak{S}_{f}\left(\mathbb{D}_{r}\right) \subset \varphi_{2}(\mathbb{D}) \quad(0 \leq r<R) .
$$

The result is sharp for the function $f_{n}$ defined in (4). At $z=-R$

$$
\left|\frac{z f_{n}^{\prime}(z)}{f_{n}(z)}\right|=\left|\left(\frac{1-(2 n+4) R-(2 n+1) R^{2}}{1-R^{2}}\right)\right|=\frac{1}{3}=\varphi_{2}(-1) \in \partial_{c} \varphi_{2}(\mathbb{D}) .
$$

9. The number $R=R_{S_{\text {cos }}^{*}}$ is the root (least positive) of the equation

$$
1-(2 n+1) R^{2}-(2 n+4) R=\cos (1)\left(1-R^{2}\right),
$$

Clearly, the values of the function

$$
\Xi_{2}(r)=\frac{-(2 n+4) r}{1-r^{2}}+\frac{1-(2 n+1) r^{2}}{1-r^{2}}=\frac{1-(2 n+1) r^{2}-(2 n+4) r}{1-r^{2}},
$$

decrease for every point on that interval $[0,1)$, for all $n \in \mathbb{N}$. As a result, for $0 \leq r<R$, $\Xi_{2}(r)>\Xi_{2}(R)=1 / \cos (1)$ and hence we have

$$
\frac{(2 n+4) r}{1-r^{2}}<\frac{1-(2 n+1) r^{2}}{1-r^{2}}-\cos (1)
$$

From (15) and (46), we have

$$
\left|\frac{z f^{\prime}(z)}{f(z)}-\frac{1-(2 n+1) r^{2}}{1-r^{2}}\right| \leq \frac{1-(2 n+1) r^{2}}{1-r^{2}}-\cos (1), \quad(|z| \leq r) .
$$


For $0 \leq r<R$, the function $\Lambda(r)=\frac{1-(2 n+1) r^{2}}{1-r^{2}}$ showing that $\Lambda^{\prime}(r)<0$ for every point on that interval $([0,1], \forall n \in \mathbb{N})$, and lies in the following interval

$$
[\Lambda(R), 1] \subset[\Lambda(.2), 1] \approx\left[1.0-2.0202 \times 10^{-2} n, 1\right] \subset\left(\cos (1), \frac{\cos (1)+\cosh (1)}{2}\right] .
$$

By [27] we have

$$
\left\{w \in \mathbb{C}:|w-a|<r_{a}\right\} \subset \Delta_{\cos } \subset\{w \in \mathbb{C}:|w(z)-1|<\cosh 1-1\},
$$

where $r_{a}$ is given by

$$
r_{a}= \begin{cases}a-\cos 1 & \left(\cos (1)<a \leq \frac{\cos (1)+\cosh (1)}{2}\right) \\ 1+\sin 1-a & \left(\frac{\cos (1)+\cosh (1)}{2}<a \leq \cosh (1)\right) .\end{cases}
$$

Hence

$$
\mathfrak{S}_{f}\left(\mathbb{D}_{r}\right) \subset \varphi_{3}(\mathbb{D}) \quad(0 \leq r<R) .
$$

The result is sharp for the function $f_{n}$, defined in (4). At $z=-R$, we have

$$
\left|\frac{z f_{n}^{\prime}(z)}{f_{n}(z)}\right|=\left|\left(\frac{1-(2 n+4) R-(2 n+1) R^{2}}{1-R^{2}}\right)\right|=\cos (1)=\varphi_{3}(-1) \in \partial_{c} \varphi_{3}(\mathbb{D}) .
$$

By putting $n=1$ in Theorem 1 we get the following result.

Corollary 1. For the class $\mathrm{Y}_{1}$, the following results hold:

1. For any $0 \leq \alpha<1$, the radius $R_{S^{*}(\alpha)}$ is the root (least positive) of the polynomial

$$
(3-\alpha) r^{2}+6 r-(1-\alpha)=0 .
$$

2. The $\mathcal{S}_{L}^{*}$ radius for $Y_{1}$ is

$$
R_{\mathcal{S}_{L}^{*}} \approx 0.070343
$$

3. The $\mathcal{S}_{p}^{*}$ radius for $\mathrm{Y}_{1}$ is

$$
R_{\mathcal{S}_{p}^{*}} \approx 0.080625
$$

4. The $\mathcal{S}_{e}^{*}$ radius for $\mathrm{Y}_{1}$ is

$$
R_{\mathcal{S}_{e}^{*}} \approx 0.10089
$$

5. The $\mathcal{S}_{\mathcal{c}}^{*}$ radius for $Y_{1}$ is

$$
R_{\mathcal{S}_{c}^{*}} \approx 0.10611
$$

6. The $\mathcal{S}_{\text {sin }}^{*}$ radius for $Y_{1}$ is

$$
R_{\mathcal{S}_{\text {sin }}^{*}} \approx 0.13199
$$

7. The $\mathcal{S}_{\sinh ^{-1}}^{*}$ radius for $Y_{1}$ is

$$
R_{\mathcal{S}_{\text {sinh }^{-1}}} \approx 0.13778
$$

8. The $\mathcal{S}_{N_{e}}^{*}$ radius for $Y_{1}$ is

$$
R_{\mathcal{S}_{N_{e}}^{*}} \approx 0.10611=R_{\mathcal{S}_{c}^{*}}
$$

9. The $\mathcal{S}_{\cos }^{*}$ radius for $Y_{1}$ is

$$
R_{\mathcal{S}_{\text {cos }}^{*}} \approx 0.07435 .
$$

These results are sharp for the function $f_{1}$ given by (6).

By putting $n=2$ in the Theorem 1 we get the following result. 
Corollary 2. For the class $\mathrm{Y}_{2}$, the following results hold:

1. The $\mathcal{S}^{*}(\alpha)$ radius for $\mathrm{Y}_{2}$ is

$$
R_{\mathcal{S}^{*}(\alpha)}=\frac{1-\alpha}{4+\sqrt{16+(1-\alpha)(5-\alpha)}} .
$$

2. The $\mathcal{S}_{L}^{*}$ radius for $\mathrm{Y}_{2}$ is

$$
R_{\mathcal{S}_{L}^{*}} \approx 0.053038
$$

3. The $\mathcal{S}_{p}^{*}$ radius for $\mathrm{Y}_{2}$ is

$$
R_{\mathcal{S}_{p}^{*}} \approx 0.060445
$$

4. The $\mathcal{S}_{e}^{*}$ radius for $\mathrm{Y}_{2}$ is

$$
R_{\mathcal{S}_{e}^{*}} \approx 0.075697
$$

5. The $\mathcal{S}_{c}^{*}$ radius for $\mathrm{Y}_{2}$ is

$$
R_{\mathcal{S}_{c}^{*}} \approx 0.079634
$$

6. The $\mathcal{S}_{\text {sin }}^{*}$ radius for $\mathrm{Y}_{2}$ is

$$
R_{\mathcal{S}_{\text {sin }}^{*}} \approx 0.099225
$$

7. The $\mathcal{S}_{\text {sinh }^{-1}}^{*}$ radius for $\mathrm{Y}_{2}$ is

$$
R_{\mathcal{S}_{\text {sinh }^{* 1}}} \approx 0.10362
$$

8. The $\mathcal{S}_{N_{e}}^{*}$ radius for $\mathrm{Y}_{2}$ is

$$
R_{\mathcal{S}_{N_{e}}^{*}} \approx 0.079634=R_{\mathcal{S}_{c}^{*}}
$$

9. The $\mathcal{S}_{\cos }^{*}$ radius for $\mathrm{Y}_{2}$ is

$$
R_{\mathcal{S}_{\text {cos }}^{*}} \approx 0.055731 \text {. }
$$

These results are sharp for the function $f_{2}$ given by (7). By putting $n=3$ in the Theorem 1 we get the following result.

Corollary 3. For the class $\mathrm{Y}_{3}$, the following results holds

1. The $\mathcal{S}^{*}(\alpha)$ radius for $\mathrm{Y}_{3}$ is

$$
R_{\mathcal{S}^{*}(\alpha)}=\frac{2(1-\alpha)}{10+\sqrt{100+4(1-\alpha)(2 n+1-\alpha)}} .
$$

2. The $\mathcal{S}_{L}^{*}$ radius for $\mathrm{Y}_{3}$ is

$$
R_{\mathcal{S}_{L}^{*}} \approx 0.042427
$$

3. The $\mathcal{S}_{p}^{*}$ radius for $\mathrm{Y}_{3}$ is

$$
R_{\mathcal{S}_{p}^{*}} \approx 0.048473
$$

4. The $\mathcal{S}_{e}^{*}$ radius for $\mathrm{Y}_{3}$ is

$$
R_{\mathcal{S}_{e}^{*}}=0.060763 .
$$

5. The $\mathcal{S}_{c}^{*}$ radius for $\mathrm{Y}_{3}$ is

$$
R_{\mathcal{S}_{c}^{*}} \approx 0.063941
$$

6. The $\mathcal{S}_{\sin }^{*}$ radius for $\mathrm{Y}_{3}$ is

$$
R_{\mathcal{S}_{\text {sin }}^{*}}=0.079791
$$

7. The $\mathcal{S}_{\sinh ^{-1}}^{*}$ radius for $\mathrm{Y}_{3}$ is

$$
R_{\mathcal{S}_{\text {sinh }^{-1}}^{*}}=0.083356
$$


8. The $\mathcal{S}_{N_{e}}^{*}$ radius for $\mathrm{Y}_{3}$ is

$$
R_{\mathcal{S}_{N_{e}}^{*}} \approx 0.063941=R_{\mathcal{S}_{c}^{*}}
$$

9. The $\mathcal{S}_{\cos }^{*}$ radius for $Y_{3}$ is

$$
R_{\mathcal{S}_{\text {cos }}^{*}}=0.044579 .
$$

These results are sharp for the function $f_{3}$ given by (8).

\section{Concluding Remarks and Observations}

In our present investigation, we have calculated radius constants for certain classes of star-like functions related with different types of domains, including, for example, the star-like functions connected with the exponential functions, the lemniscate of Bernoulli, sine functions, cardioid functions, sine hyperbolic inverse functions, Nephroid functions, cosine functions and parabolic star-like functions. We have, then, shown that the results presented in this paper are sharp. Additionally, by assigning different values to some perpetrators we have obtained some interesting and remarkable special cases in form of corollaries.

In concluding our current investigation, we would like to bring to the attention of interested readers the possibility of studying fundamental or quantum (or q-) generalizations of the results we have elaborated here. The interested readers may also use iteration processes to develop an approximate common fixed point of the mapping $\frac{z f^{\prime}(z)}{f(z)}$ (see, for details, $[28,29])$. Srivastava's recently published survey-cum-expository review study [30] also shows that [31] has impacted and driven this research area. However, as Srivastava (see [30], p. 340 and [32], Section 5, pp. 1511-1512) has previously proved the $(\mathfrak{p}, \mathfrak{q})$-variations of the intended $\mathfrak{q}$-results because the forced-in parameter $\mathfrak{p}$ is insignificant, it will lead to insubstantial work. Furthermore, in light of Srivastava's more recent article [32], the interested reader should be advised not to be misled to believe that the so-called $k$-Gamma function provides a "generalization" of the classical (Euler's) Gamma function. Similar remarks will apply, also, to all of the usages of the so-called $k$-Gamma function including (for example) the so-called $(k, s)$-extensions of the Riemann-Liouville integral and other operators of fractional integral and fractional derivatives.

Author Contributions: Conceptualization, M.H., M.A. and N.K.; methodology, C.Z., M.A. and K.A.; software, N.K. and B.K.; validation, C.Z., M.H. and M.A.; formal analysis, M.A. and N.K.; investigation, C.Z., M.H., K.A. and B.K.; resources, M.A. and N.K.; writing-original draft preparation, M.H., K.A. and B.K.; writing — review and editing, B.K.; visualization, C.Z. and K.A.; supervision, M.A. and N.K.; funding acquisition, C.Z. and B.K. All authors have read and agreed to the published version of the manuscript.

Funding: This research received no external funding.

Acknowledgments: The authors are grateful to the editor and the reviewers for their valuable comments and suggestions.

Conflicts of Interest: The authors declare no conflict of interest.

\section{References}

1. Grunsky, H. Neue abschätzungen zur konformen abbildung ein-und mehrfachzusammenhngender bereiche. Schr. Dtsch. Math. Ver. 1934, 43, 140-143.

2. Reade, M.O. On close-to-convex univalent functions. Mich. Math. 1955, 3, 59-62. [CrossRef]

3. Shi, L.; Srivastava, H.M.; Khan, M.G.; Khan, N.; Ahmad, B.; Khan, B.; Mashwani, W.K. Certain Subclasses of Analytic Multivalent Functions Associated with Petal-Shape Domain. Axioms 2021, 10, 291. [CrossRef]

4. Ebadian, A.; Cho, N.E.; Adegani, E.A.; Yalçın, S. New Criteria for Meromorphic star-likeness and Close-to-Convexity. Mathematics 2020, 8, 847. [CrossRef]

5. Naeem, M.; Hussain, S.; Mahmood, T.; Khan, S.; Darus, M. A New Subclass of Analytic Functions Defined by Using Salagean $q$-Differential Operator. Mathematics 2019, 7, 458. [CrossRef]

6. Liu, L.; Liu, J.-L. Properties of Certain Multivalent Analytic Functions Associated with the Lemniscate of Bernoulli. Axioms 2021, 10, 160. [CrossRef] 
7. Soybaş, D.; Joshi, S.B.; Pawar, H. On a Certain Subclass of Analytic Functions Involving Integral Operator Defined by Polylogarithm Function. Mathematics 2019, 7, 66. [CrossRef]

8. Wang, B.; Srivastava, R.; Liu, J.-L. A Certain Subclass of Multivalent Analytic Functions Defined by the $q$-Difference Operator Related to the Janowski Functions. Mathematics 2021, 9, 1706. [CrossRef]

9. Shi, L.; Khan, M.G.; Ahmad, B.; Mashwani, W.K.; Agarwal, P.; Momani, S. Certain Coefficient Estimate Problems for Three-LeafType Starlike Functions. Fractal Fract. 2021, 5, 137. [CrossRef]

10. Islam, S.; Khan, M.G.; Ahmad, B.; Arif, M.; Chinram, R. q-Extension of Starlike Functions Subordinated with a Trigonometric Sine Function. Mathematics 2020, 8, 1676. [CrossRef]

11. MacGregor, T.H. The radius of univalence of certain analytic functions. Proc. Amer. Math. Soc. 1963, 14, 514-520. [CrossRef]

12. MacGregor, T.H. The radius of univalence of certain analytic functions II. Proc. Amer. Math. Soc. 1963, 14, 521-524. [CrossRef]

13. Ratti, J.S. The radius of convexity of certain analytic functions. Indian J. Pure Appl. Math. 1970, 1, 30-36. [CrossRef]

14. Lecko, A.; Sim, Y.J. Coefficient problems in the subclasses of close-to-star functions. Results Math. 2019, 3, 104. [CrossRef]

15. Sokól, J.; Stankiewicz, J. Radius of convexity of some subclasses of strongly starlike functions. Zesz. Nauk. Politech. Rzesz. Math. 1996, 19, 101-105.

16. Kocur, M.F. Radii of starlikeness and of convexity in some classes of analytic functions in the disk. Mat. Zametki 1979, 25, 675-679.

17. Sharma, K.; Jain, N.K.; Ravichandran, V. Starlike functions associated with a cardioid. Afr. Mat. 2016, 27, 923-939. [CrossRef]

18. Ali, R.M.; Jain, N.K.; Ravichandran, V. On the radius constants for classes of analytic functions. Bull. Malays. Math. Sci. Soc. 2013, $36,23-38$.

19. Wani, L.A.; Sawaminathan, A. Starlike and convex functions associated with a nephroid domain. Bull. Malays. Math. Sci. Soc. 2021, 44, 79-104. [CrossRef]

20. Cho, N.E.; Kumar, V.; Kumar, S.S.; Ravichandran, V. Radius problems for starlike functions associated with the sine function. Bull. Iran. Math. Soc. 2019, 45, 213-232. [CrossRef]

21. Mendiratta, R.; Nagpal, S.; Ravichandran, V. A subclass of starlike functions associated with left-half of the lemniscate of Bernoulli. Internat. J. Math. 2014, 25, 1450090. [CrossRef]

22. Gangadharan, A.; Ravichandran, V.; Shanmugam, T.N. Radii of convexity and strong starlikeness for some classes of analytic functions. J. Math. Anal. Appl. 1997, 211, 301-313. [CrossRef]

23. Ali, R.M.; Jain, N.K.; Ravichandran, V. Radii of starlikeness associated with the lemniscate of Bernoulli and the left-half plane. Appl. Math. Comput. 2012, 128, 6557-6565. [CrossRef]

24. Ma, W.C.; Minda, D. Uniformly convex functions. Ann. Polon. Math. 1992, 57, 165-175. [CrossRef]

25. Mendiratta, R.; Nagpal, S.; Ravichandran, V. On a subclass of strongly starlike functions associated with exponential function. Bull. Malays. Math. Sci. Soc. 2015, 38, 365-386. [CrossRef]

26. Kumar, S.; Arora, K. Starlike Functions associated with a Petal Shaped Domain. arXiv 2020, arXiv:2010.10072.

27. Bano, K.; Raza, M. Starlike functions associated with cosine functions. Bull. Iran. Math. Soc. 2021, 47, 1513-1532. [CrossRef]

28. Garodia, C.; Abdou, A.A.N.; Uddin, I. A New Modified Fixed-Point Iteration Process. Mathematics 2021, 9, 3109. [CrossRef]

29. Jantschi, L.; Balint, D.; Bolboaca, S.D. Multiple Linear Regressions by Maximizing the Likelihood under Assumption of Generalized Gauss-Laplace Distribution of the Error. Comput. Math. Methods Med. 2016, 2016, 8578156. [CrossRef]

30. Srivastava, H.M. Operators of basic (or $q$-) calculus and fractional $q$-calculus and their applications in geometric function theory of complex analysis. Iran. J. Sci. Technol. Trans. A Sci. 2021, 44, 327-344. [CrossRef]

31. Srivastava, H.M.; Wanas, A.K.; Srivastava, R. Applications of the $q$-Srivastava-Attiya operator involving a certain family of bi-univalent functions associated with the Horadam polynomials. Symmetry 2021, 13, 1230. [CrossRef]

32. Srivastava, H.M. Some parametric and argument variations of the operators of fractional calculus and related special functions and integral transformatioons. J. Nonlinear Convex Anal. 2021, 22, 1501-1520. 